\title{
Towards a Vortex Generator Model for Integral Boundary Layer Methods
}

\author{
Akshay Koodly Ravishanara*† \\ ECN part of TNO, Petten, 1755 LE The Netherlands \\ University of Twente, 7500 AE, Enschede, The Netherlands \\ Huseyin Özdemir \\ ECN part of TNO, Petten, 1755 LE, The Netherlands \\ Andrea Franco ${ }^{\S}$ \\ University of Padova, Padova 35122 Italy
}

\begin{abstract}
In this study a new approach to model the effect of vortex generators in integral boundary layer equations is described. Vortex generators (VGs) are commonly used on wind turbine blades to avoid early separation of flow which helps not only to increase the lift but also delays the stall. Delaying the stall angle of wind turbine blades will allow for operation over larger range of angle of attacks and thus increase power production from wind turbines. Typically, VGs are submerged in the boundary layer and generate vortices that mix high-energy fluid from outer flow with the slow-moving boundary layer. Within this study the effect of the VGs on the 2-D boundary layers are modeled as additional mixing. The presence of a new shear layer will lead to additional viscous dissipation and affect the wall shear stress. To model these effects new terms are introduced into the integral boundary layer equations to account for the extra mass and momentum flux and turbulence production. The additional unknowns introduced into the system of equations are derived using CFD simulations. Initial CFD simulations on a flat plate with body fitted meshes around VGs are used to obtain an algebraic model. The new model is implemented in an aerodynamic analysis and design tool and preliminary results are presented. The theory developed here will be extended for flows over airfoils.
\end{abstract}

\section{Nomenclature}

$C_{D} \quad$ Dissipation coefficient

$C_{d} \quad$ Drag coefficient

$C_{f} \quad$ Skin friction coefficient

$C_{l} \quad$ Lift coefficient

$C_{p} \quad$ Pressure coefficient

$C_{\tau} \quad$ Shear stress coefficient

$h \quad$ height of the $\mathrm{VG}$

$P \quad$ Pressure

$U_{c} \quad$ Characteristic convection velocity $u_{e} \quad$ Edge velocity

$U_{h} \quad$ Velocity at the upper side of the mixing region

$U_{l} \quad$ Velocity at the lower side of the mixing region

$U_{s} \quad$ Characteristic velocity difference

$u_{*} \quad$ Friction velocity

$V G \quad$ Vortex generator

$x / h \quad \frac{X-X_{V G}}{h}$, Non dimensional location based on distance from the VG and height of VG

$X_{V G} \quad x$ - coordinate of the VG

$y^{+} \quad$ Non-dimensional length scale

c Chord length

\footnotetext{
*Junior Researcher, Wind Energy, Westerduinweg 3, akshay.koodlyravishankara@tno.nl, Member AIAA

${ }^{\dagger}$ PhD Candidate, Engineering Fluid Dynamics Group, a.koodlyravishankara@utwente.nl

$\doteqdot$ Researcher, Wind Energy, Westerduinweg 3, huseyin.ozdemir@tno.nl, Member AIAA

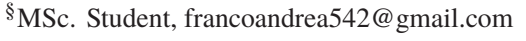




\section{Subscripts}

bl Sum of the effects of wall and outer regions

eff Effective quantity throughout the boundary layer

$E Q \quad$ Value at the equilibrium condition

$m \quad$ Contribution from the mixing region

$o \quad$ Contribution from the outer region

$V G \quad$ Value at the location of the vortex generator

$w \quad$ Contribution from the wall region
Symbols

$\delta \quad$ Boundary layer thickness

$\delta^{*} \quad \int_{0}^{\delta}\left(1-\frac{u}{u_{e}}\right) d y$, Displacement thickness

$\delta^{k} \quad \int_{0}^{\delta} \frac{u}{u_{e}}\left(1-\frac{u^{2}}{u_{e}^{2}}\right) d y$, Energy thickness

$\tau \quad$ Shear stress

$\tau_{w} \quad$ Wall shear stress

$\theta \quad \int_{0}^{\delta} \frac{u}{u_{e}}\left(1-\frac{u}{u_{e}}\right) d y$, Momentum thickness

\section{Introduction}

As the threat of global warming becomes imminent, there is an urgent need for sustainable sources of energy like wind power to meet larger power demands. While larger turbines are being designed to meet the new demands, there is a considerable effort to improve the efficiency of (existing) wind turbines as well. Many innovative concepts have been used and the use of flow enhancement devices or blade add-ons are becoming more common. An example of such a device is the vortex generator (VG). The concept of vortex generator is quite old and there has been a considerable research into analyzing the effects of vortex generators[1,2] Vortex generators energize the boundary layer and allow for the boundary layer to remain attached for longer[3,4]. Vortex generators have been successfully used to increase lift produced by airfoil sections and delay stall[5]. Prior research on modeling VGs in CFD methods can be found in references, e.g. [6] and [7]. However, the above mentioned modeling methods require an expensive CFD simulations. Performing such (three-dimensional) CFD simulations during the design phase of wind turbines is prohibitively expensive and a simpler solution is desired. Current wind turbine design tools are based on Blade Element Momentum (BEM) theory[8] and other local blade aerodynamic methods based on the solution of integral boundary layer equations together with the inviscid potential equations coupled with an appropriate viscous-inviscid interaction scheme[9-11] (also called interacting boundary layer method). Incorporating the effects of blade add-ons like VGs into such tools will allow for better and more efficient designs at a fraction of the cost compared to full three-dimensional CFD methods.

While the vortices induced by the VGs are three dimensional in nature, in this study, an approximate model that can capture the effects of VGs (e.g. on lift prediction) on a two-dimensional interacting boundary layer method is sought. To this end, the mixing induced by the vortices will be modeled by a mixing layer that interacts with the boundary layer. The integral boundary layer method is based on boundary layer theory which states that the effect of viscosity is confined to a small region around the body (boundary layer) and further away from the body flow is largely inviscid. The Navier-Stokes equations can thus be simplified for the two regions with matching solutions at the interface, allowing for faster computational times. The flow domain away from the body can be described by the inviscid Euler equations and can be approximated by using e.g. panel method. The boundary layer equations in the vicinity of the body can be derived from Navier-Stokes equations under the limit of large Reynolds numbers. The boundary layer equations can then be further simplified by integrating along the wall normal direction to obtain the integral boundary layer equations. In this study we present a new model for VGs based on the derivation of the two dimensional boundary layer equations including an integration also accounting for the presence of the vortex generator.

In the section III the boundary layer separation is examined for laminar (III.A) and turbulent flows(III.B). Section III.C will present the ideas and theory behind the model. Section IV presents a detailed derivation of the proposed model. The boundary layer profiles extracted from the CFD simulations of flow over a flat plate with and without VGs and are used to derive an algebraic model for the IBL method and presented in VII. The flat plate models are implemented in an in-house integral boundary layer method[11]. 


\section{Boundary layer separation}

\section{A. Laminar boundary layer}

Boundary layer is a very interesting and complicated region of the flow field. Due to the no-slip condition at the wall, the incoming flow slows down and the velocities approach to zero at the wall and gradually increases until it matches the edge velocity, $u_{e}$, at the edge of the boundary layer (approximately the free-stream velocity). The distance from the wall to the edge of the boundary layer is known as the boundary layer thickness, $\delta$. Based on the boundary layer theory, the boundary layer equations for steady two dimensional flows can be derived starting from the non-dimensionalized Navier-Stokes equations[12] and read as follows

$$
\begin{gathered}
\frac{\partial u}{\partial x}+\frac{\partial v}{\partial y}=0, \\
\frac{\partial u^{2}}{\partial x}+\frac{\partial u v}{\partial y}=-\frac{\partial P}{\partial x}+v \frac{\partial^{2} u}{\partial y^{2}}, \\
\frac{\partial P}{\partial y}=0 .
\end{gathered}
$$

The Eq. 1 is the continuity equation which remains unchanged under the assumption of large $\operatorname{Re}$ numbers $(\operatorname{Re}>>1)$. Assuming the flow to be mostly aligned with the $x$ - direction, the $y$ - momentum equation is reduced to the condition that the normal pressure gradient is zero across the boundary layer (Eq. 3). Thus the pressure in the boundary layer is imposed on it by the flow outside the boundary layer. And this flow outside the boundary layer is governed by the inviscid Euler's equation as viscous effects become negligible. Applying the momentum equation, Eq. 2, to the edge of the boundary layer, where $v=0$ and assuming there is no mean flow gradient, we obtain a relation for pressure gradient in terms of edge velocity

$$
\frac{\partial P}{\partial x}=-u_{e} \frac{\partial u_{e}}{\partial x} .
$$

This relation is essentially the Bernoulli equation for inviscid flow along a streamline which is expected to be at the edge of the boundary layer and it is assumed to be where the flow becomes inviscid.

Depending on the external pressure gradient, the slow moving boundary layer can either remain attached or separate from the wall. If the flow starts to separate, the velocity development along the flow direction would decrease until a flow reversal occurs. In other words, $\frac{\partial u}{\partial x}$ will start decreasing until it reaches zero and then will change sign. Boundary layers are prone to separation under adverse pressure gradients (Figure. 1). Consider a simplified analysis and neglect the $y-$ component of velocity, $v$, from the momentum equations (this assumption is not far from reality in most boundary layer flows):

$$
u \frac{\partial u}{\partial x}=-\frac{\partial P}{\partial x}+v \frac{\partial^{2} u}{\partial y^{2}} .
$$

In an adverse pressure gradient $(d p>0)$, the term $-\frac{\partial P}{\partial x}$ is negative thus, only the shear stress contribution is preventing the flow from separating. Very near to the wall, the shear stress is essentially the wall stress, $\tau_{w}$ and the flow will separate once the wall stress contribution is not enough to overcome the adverse gradient. In the absence of a pressure gradient (like flat plates), $d p=0$, so, separation occurs if wall stress becomes zero $\left(\tau_{w}=0\right)$ or an inflection point appears in the boundary layer profile.

\section{B. Turbulent boundary layer}

Introduction of turbulence to flow has a significant effect on the boundary layers. Due to increased energy transfer between length scales, there is now larger energy even in the boundary layer. However, due to the no-slip condition the velocity at the wall must still go to zero which leads to an even larger gradient very near to the wall. Using the Reynolds averaging procedure, the turbulent Reynolds stresses are represented by the eddy viscosity term, $v_{t}$ and the Eq. 5 becomes

$$
u \frac{\partial u}{\partial x}=-\frac{\partial P}{\partial x}+\left(v+v_{t}\right) \frac{\partial^{2} u}{\partial y^{2}} .
$$




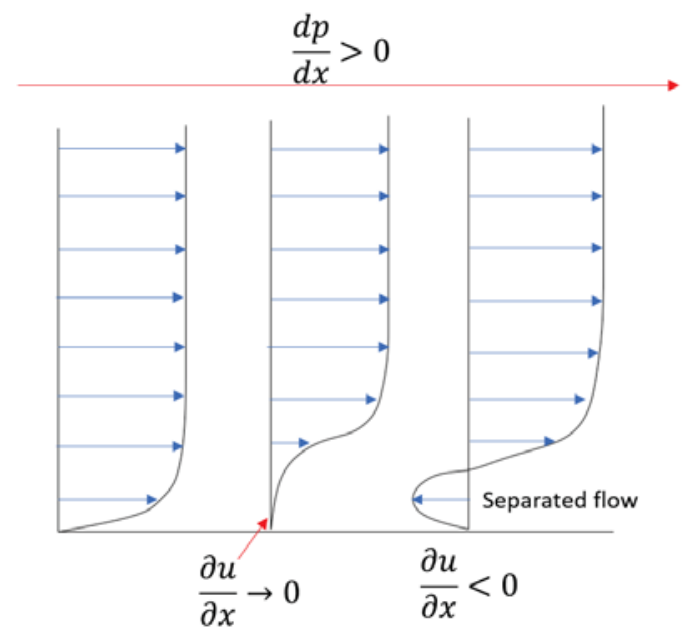

Fig. 1 A schematic visualization of the boundary layer separation.

Since the eddy viscosity is positive, it is easy to see that the turbulent flow separates later than laminar flow and can overcome larger adverse pressure gradients. This is a very simplified analysis of turbulent boundary layers and to better understand the turbulent boundary layer, a deeper insight is necessary.

The main characteristic of turbulent flows is the appearance of vortices and the energy cascade from the largest length scales to the smallest. As we approach closer to the wall, the larger scales dissipate away and only smaller scales remain. Very near to the wall, only the smallest scales remain and the viscous stresses become significant again. Hence, there are different regions within the turbulent boundary layer depending on the relative magnitudes of the Reynolds stresses and the viscous stresses. Broadly, the turbulent boundary layer can be divided into two layers, namely the inner and outer layers (Figure 2). The inner layer is the one closest to the wall and viscosity is relatively large in this region. Very near to the wall, the viscous stresses dominate and the Reynolds stresses tend to zero. As we move away from the wall the Reynolds stresses start increasing and the viscous and Reynolds stresses reach to an equilibrium. At the edge of the inner layer, the Reynolds stress are large enough to completely dominate the viscous stresses. In the outer layer of the boundary, the viscous stresses disappear and only the Reynolds stress exists. The exact demarcation between the inner and outer layer is not sharp and depends on local flow conditions and Reynolds number. In general, the outer layer spans from about $y / \delta \approx 0.1$ to the edge of the boundary layer. Within the inner layer, it is more convenient to use a different length scale instead of $\delta$ and thus viscous units are used. A velocity scale, friction velocity[13], is defined based on the wall stress as

$$
u_{*}=\sqrt{\frac{\tau_{w}}{\rho}},
$$

where, $\tau_{w}$ is the wall shear stress and $\rho$ is the density.

Based on the friction velocity, a non-dimensional length scale, $y^{+}$can be defined as

$$
y^{+}=\frac{y u_{*}}{v} .
$$

The inner layer is further subdivided into different regions based on $y^{+}$. The different regions within a turbulent boundary layer is shown in Figure. 2[13].

\section{Vortex generator in the boundary layer}

The VG has an analogous effect on turbulent boundary layers as the introduction of turbulence has on laminar boundary layers[2]. As the name suggests, a vortex generator generates vortices that entrain high energy fluid from outside of the boundary layer and mix it with the boundary layer flow. The additional momentum and energy introduced to the boundary layer helps to overcome more severe adverse pressure gradients and the boundary layer can remain attached for longer[2]. 


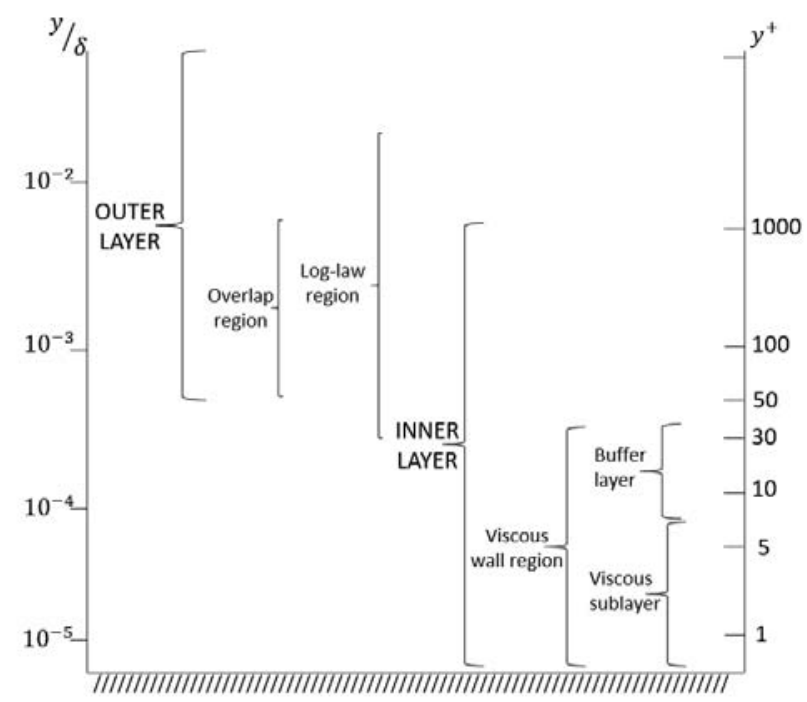

Fig. 2 An overview of wall regions defined in terms of $y^{+}$and $y / \delta$ at $\operatorname{Re}=10^{6}[13]$.

The vortex generator is assumed to be submerged in the boundary layer (low-profile VG[1]) but extend well into the outer layer of the boundary layer (see III.B). For instance, the height of the vortex generators used in experiments typically range from $5 \mathrm{~mm}$ [5] to about $10 \mathrm{~mm}$ [14]. From the operational Reynolds numbers in the experiments, one can compute the height of the vortex generators in wall units to be approximately between $y^{+}=1000$ and $y^{+}=2050$ which is far away from the regions where viscous stresses from the wall are dominant.

However, the additional shear layer introduced due to mixing will now create a new region within the boundary layer where viscous stresses become significant again away from the wall. Unlike a fully turbulent boundary layer where the outer layer is dominated by only Reynolds stresses, the VG will introduce additional mean flow gradients and viscous stresses in the outer layer as well which will also lead to an increase in the production of turbulent kinetic energy within the boundary layer.

In essence, the underlying assumption of the model is that the presence of mixing induced by VGs will increase viscous dissipation due to the additional mean flow gradients. The new eddy viscosity, $v_{t}^{\prime}$ (which is different than $v_{t}$ ), will also increase due to additional production of turbulent kinetic energy. Thus the extra viscous and eddy dissipation will lead to a more controlled rate of growth of the boundary layer and delay separation. This can also be verified by looking at the simplified relations in Eqs. 5 and 6. If the VG were to introduce an additional term to counter the pressure gradient, we have,

$$
u \frac{\partial u}{\partial x}=-\frac{\partial P}{\partial x}+\left(v+v_{t}^{\prime}\right) \frac{\partial^{2} u}{\partial y^{2}}+\text { viscous dissipation due to VG. }
$$

The additional viscous dissipation and a larger $v_{t}^{\prime}$ can help the boundary layer to overcome an even larger adverse pressure gradient and remain attached for longer than the turbulent boundary layer (Eq. 6). In the following sections, it will be shown that indeed an extra dissipation term arises due to the VG and its exact form will be derived. To derive the model it is assumed that the effect of the VG is confined mostly to the outer layer (referred to as the mixing region and denoted by $m$ ) and has very little effect on the flow very near to the wall (referred to as the wall region and denoted by $w$ ).

\section{Modified IBL Equations}

The integral boundary layer (IBL) equations are obtained by taking the zeroth and the first moment of the two dimensional boundary layer equations. The $n^{t h}$ moment of the boundary layer equation is defined as[15]

$$
\text { [Eq. 2] } \times(n+1) u^{n}-\left[\text { Eq. 1] } \times\left(u_{e}^{n+1}-u^{n+1}\right)\right. \text {. }
$$


Integrating the continuity equation along the total boundary layer thickness, from $y=0$ to $y=\delta$, and using the no-slip condition at the wall we get,

$$
\left.v\right|_{y=\delta}=-\int_{0}^{\delta} \frac{\partial u}{\partial x} d y
$$

\section{A. Momentum integral equation}

Integrate the $x$-momentum equation between $y=0$ and $y=\delta$,

$$
\int_{0}^{\delta}\left(\frac{\partial u^{2}}{\partial x}+\frac{\partial u v}{\partial y}+\frac{\partial P}{\partial x}\right) d y=\int_{0}^{\delta}\left(v \frac{\partial^{2} u}{\partial y^{2}}\right) d y
$$

Integrate the momentum equation and using Eqs. 5 and 9,

$$
\frac{\partial}{\partial x} \int_{0}^{\delta}\left(u^{2}-u u_{e}\right) d y+\frac{\partial u_{e}}{\partial x} \int_{0}^{\delta}\left(u-u_{e}\right) d y=\int_{0}^{\delta}\left(v \frac{\partial^{2} u}{\partial y^{2}}\right) d y .
$$

Here, the variation of edge velocity is assumed to be independent of the integral.

At this point, we introduce our assumptions for modeling the effect of the vortex generators. Along the wall normal direction, we assume the wall effect is prevalent up to $y=\delta_{w}$ and from $y=\delta_{w}$ to $\delta_{m}$, the mixing dominates. The boundary layer extends further up to $y=\delta$. The velocity $u$ is assumed to be composed of: $u_{w}$, the velocity due to the wall effects, $u_{m}$, the velocity due to mixing effect and $u_{o}$, the velocity in the outer region outside the influence of mixing region extending up to the edge of the boundary layer:

$$
u= \begin{cases}u_{w}, & 0<y<\delta_{w}, \\ u_{m}, & \delta_{w}<y<\delta_{m}, \\ u_{o}, & \delta_{m}<y<\delta .\end{cases}
$$

The integral can now be split into three parts as follows

$$
\begin{array}{r}
\frac{\partial}{\partial x} \int_{0}^{\delta_{w}}\left(u_{w}^{2}-u_{w} u_{e}\right) d y+\frac{\partial}{\partial x} \int_{\delta_{w}}^{\delta_{m}}\left(u_{m}^{2}-u_{m} u_{e}\right) d y+\frac{\partial}{\partial x} \int_{\delta_{m}}^{\delta}\left(u_{o}^{2}-u_{o} u_{e}\right) d y \\
+\frac{\partial u_{e}}{\partial x} \int_{0}^{\delta_{w}}\left(u_{w}-u_{e}\right) d y+\frac{\partial u_{e}}{\partial x} \int_{\delta_{w}}^{\delta_{m}}\left(u_{m}-u_{e}\right) d y+\frac{\partial u_{e}}{\partial x} \int_{\delta_{m}}^{\delta}\left(u_{o}-u_{e}\right) d y \\
=\int_{0}^{\delta_{w}}\left(v \frac{\partial^{2} u_{w}}{\partial y^{2}}\right) d y+\int_{\delta_{w}}^{\delta_{m}}\left(v \frac{\partial^{2} u_{m}}{\partial y^{2}}\right) d y+\int_{\delta_{m}}^{\delta}\left(v \frac{\partial^{2} u_{o}}{\partial y^{2}}\right) d y
\end{array}
$$

Using the definitions of displacement thickness $\delta^{*}$ and momentum thickness $\theta$ Eq. 12 becomes,

$$
-\frac{\partial\left(u_{e}^{2} \theta_{e f f}\right)}{\partial x}-\left(u_{e} \delta_{e f f}^{*}\right) \frac{\partial u_{e}}{\partial x}=\int_{0}^{\delta_{w}}\left(v \frac{\partial^{2} u_{w}}{\partial y^{2}}\right) d y+\int_{\delta_{w}}^{\delta_{m}}\left(v \frac{\partial^{2} u_{m}}{\partial y^{2}}\right) d y+\int_{\delta_{m}}^{\delta}\left(v \frac{\partial^{2} u_{m}}{\partial y^{2}}\right) d y .
$$

Here $\theta_{\text {eff }}$ and $\delta_{\text {eff }}^{*}$ are defined as

$$
\begin{aligned}
& \theta_{e f f}=\left(\theta_{b l}+\theta_{m}\right), \\
& \delta_{e f f}^{*}=\left(\delta_{b l}^{*}+\delta_{m}^{*}\right) .
\end{aligned}
$$

$\delta_{b l}^{*}$ and $\theta_{b l}$ are the displacement and momentum thicknesses due to the sum of the wall region and the outer layer and $\delta_{m}^{*}$ and $\theta_{m}$ are due to mixing. In a boundary layer, without any VG, the mixing contributions will be zero and the sum of contributions from the wall and outer layers are generally combined into one thickness.

The Eq. 13 is very similar to the standard integral momentum equation. Integrating the right-hand-side (RHS) of this equation can also be reduced to the standard form as follows

$$
\left.v \frac{\partial u_{w}}{\partial y}\right|_{y=\delta_{w}}-\left.v \frac{\partial u_{w}}{\partial y}\right|_{y=0}+\left.v \frac{\partial u_{m}}{\partial y}\right|_{y=\delta_{m}}-\left.v \frac{\partial u_{m}}{\partial y}\right|_{y=\delta_{w}}+\left.v \frac{\partial u_{o}}{\partial y}\right|_{y=\delta}-\left.v \frac{\partial u_{o}}{\partial y}\right|_{y=\delta_{m}}
$$


Naturally, the intermediate terms all cancel out leaving only the terms at the wall and the edge of the boundary layer and the integrand at $y=\delta$ approaches to zero as there are no normal velocity gradients outside the boundary layer leaving

$$
\text { RHS }=-\left.v \frac{\partial u_{w}}{\partial y}\right|_{y=0}=\frac{\tau_{w a l l}}{\rho},
$$

where $\tau_{\text {wall }}$ is the wall shear stress and is given by

$$
\tau_{w a l l}=-\left.\mu \frac{\partial u}{\partial y}\right|_{y=\delta}
$$

with, $\mu$ is dynamic viscosity coefficient. Thus, the RHS reduces to the same form as the standard IBL equations irrespective of the presence of the VG. This is expected as integration of flow equations accounts only for the global effects and the internal flow details do not appear in the equations. However, the wall stress, $\tau_{w a l l}$ is unknown. The skin friction coefficient is defined as

$$
C_{f}=\frac{\tau_{w a l l}}{1 / 2 \rho u_{e}^{2}}
$$

\section{B. Kinetic energy integral equation}

The kinetic energy integral equation is obtained by taking the first moment of the boundary layer equations where the $n^{\text {th }}$ moment is defined as (Eq. 8)

Thus, the first moment is

$$
2 u^{2} \frac{\partial u}{\partial x}+2 u v \frac{\partial u}{\partial y}-2 u u_{e} \frac{\partial u_{e}}{\partial x}-\left(u_{e}^{2}-u^{2}\right) \frac{\partial u}{\partial x}-\left(u_{e}^{2}-u^{2}\right) \frac{\partial v}{\partial y}=2 u v \frac{\partial^{2} u}{\partial y^{2}},
$$

Integration along the wall normal direction from $y=0$ to $y=\delta$,and using Eq. 11 similar to the momentum integral equation leads to

$$
\frac{\partial u_{e}^{3} \delta_{e f f}^{k}}{\partial x}=\int_{0}^{\delta_{w}} 2 u_{w} v \frac{\partial^{2} u_{w}}{\partial y^{2}} d y+\int_{\delta_{w}}^{\delta_{m}} 2 u_{m} v \frac{\partial^{2} u_{m}}{\partial y^{2}} d y+\int_{\delta_{m}}^{\delta} 2 u_{o} v \frac{\partial^{2} u_{o}}{\partial y^{2}} d y
$$

Once again, the intermediate terms cancel out leaving the RHS in the same form as the standard integral boundary layer equation model. The dissipation coefficient can thus be defined as

$$
\begin{array}{r}
D=\int_{0}^{\delta} 2 \tau \frac{\partial u}{\partial y} d y \\
C_{D}=\frac{D}{1 / 2 \rho u_{e}^{3}} .
\end{array}
$$

To summarize, the modified integral boundary layer equations can be written for laminar flows as follows:

$$
\begin{aligned}
\frac{\partial\left(u_{e}^{2} \theta_{e f f}\right)}{\partial x}+\left(u_{e} \delta_{e f f}^{*}\right) \frac{\partial u_{e}}{\partial x} & =\frac{C_{f}}{2} u_{e}^{2} \\
\frac{\partial u_{e}^{3} \delta_{e f f}^{k}}{\partial x} & =C_{D} u_{e}^{3}
\end{aligned}
$$

together with the following new variables,

$$
\begin{aligned}
& \theta_{\text {eff }}=\left(\theta_{b l}+\theta_{m}\right), \\
& \delta_{\text {eff }}^{*}=\left(\delta_{b l}^{*}+\delta_{m}^{*}\right), \\
& \delta_{\text {eff }}^{k}=\left(\delta_{b l}^{k}+\delta_{m}^{k}\right) .
\end{aligned}
$$


As it can be seen from the above equations, there are more unknowns than the number of equations $\left(\theta, \delta^{*}, \delta^{k}, C_{f}, C_{D}\right)$ thus this system of equations can be closed by closure relations. $C_{f}$ and $C_{D}$ are represented in terms of $H, H_{k}$ and $\operatorname{Re}_{\theta}$ and these closure relations can be derived using parametric velocity profile families like Falkner-Skan flows $[9,16]$.

In a turbulent boundary layer the velocity can be split using the Reynolds decomposition as

$$
u=\bar{u}+u^{\prime}
$$

where $\bar{u}$ is the mean or time averaged component and $u^{\prime}$ is the fluctuating component. The momentum and kinetic energy integral equations can be derived by following the same procedure outlined above for the time averaged equations. As discussed earlier in section III.B additional Reynolds stresses appear in the boundary layer and thus, the shear stress is now,

$$
\tau=\mu \frac{\partial u}{\partial y}-\rho \overline{u^{\prime} v^{\prime}} .
$$

In turbulent boundary layers, a new non dimensional quantity, $C_{\tau}$, the maximum Reynolds shear stress coefficient is introduced as,

$$
C_{\tau}=\frac{1}{u_{e}^{2}}\left(-\overline{u^{\prime} v^{\prime}}\right) .
$$

Additional closures for $C_{\tau}$ are needed as this term does not exist in laminar flows. Also, skin friction coefficient, $C_{f}$, and dissipation coefficient, $C_{D}$, are both defined using the shear stress and the addition of turbulence will require a modification to the closures defined earlier. This is also apparent as the velocity profiles in the turbulent boundary layer is different from those assumed during the derivation for laminar boundary layer closures. To derive turbulent closures, a composite velocity profile consisting of the velocity relations $([13,16])$ in different regions of the turbulent boundary layer (Fig. 2) are used. However such profiles assume only a local dependence of boundary layer parameters on Reynolds stresses. To account for upstream history effects another equation known as the shear lag equation $[9,10]$ is added to the system of equations. The local shear stress is obtained by solving a shear-stress transport equation. A parameter, equilibrium shear stress coefficient, $C_{\tau_{E Q}}$, is defined as the value of the shear stress coefficient which would occur if the local boundary layer was part of an equilibrium flow $[9,16]$. The shear lag equation is defined as

$$
\frac{\delta}{C_{\tau}} \frac{\partial C_{\tau}}{\partial x}=K_{C}\left(C_{\tau_{E Q}}^{1 / 2}-C_{\tau}^{1 / 2}\right)
$$

The closure relations definitions are skipped for the sake of brevity and can be found in numerous references like $[9,10,16]$.

In a similar vein, to derive closure relations to account for the effect of VG, new parametric definitions of velocity profiles are needed. In section V, the velocity profiles for boundary layers for flows with and without VG are derived using CFD simulations. As expected based on the discussion in section III.C, the introduction of a VG will lead to the formation of a mixing layer (like Fig. 3) within the boundary layer and thus change the boundary layer parameters (at least locally around the VG). Thus, new parametric definitions for velocity profiles can be obtained based on mixing theory which can subsequently be used to derive the new closure relations.

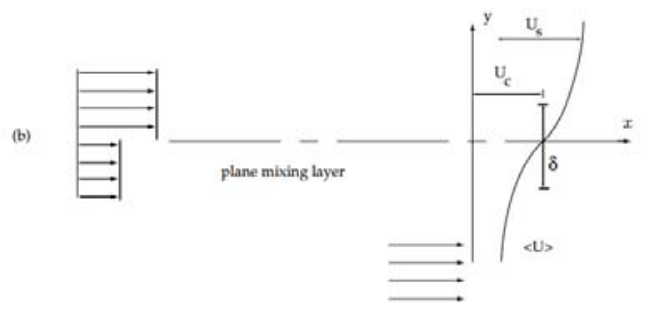

(a) An example of a plane mixing layer.

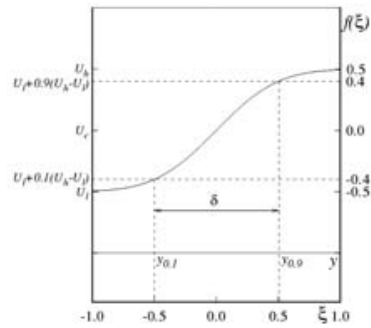

(b) Example of a scaled velocity profile in a mixing layer.

Fig. 3 A plane mixing layer[13]. 


\section{CFD results}

CFD simulations for flow over flat plate are carried out using an open source tool SU2[17] which is a compressible flow solver with a preconditioning and an artificial compressibility options for low Mach number flows (essentially incompressible) as in the current case.

In this study, the experimental study of Baldacchino [18] is replicated where the VG is placed on a flat plate as shown in Fig.4a. CFD simulations are performed for a flow with laminar to turbulent transition using BC transition [19] and Spalarat-Allamaras turbulence models for steady, incompressible flow conditions, first for no VG (clean) cases and then for cases including the VG at a Re number of $2.5 e 6$ at the VG location. The flat plate is $4.0 m$ long and the VG is placed at $X_{V G}=0.985 \mathrm{~m}$. As the aim of the study is to implement the effect of the VGs in a 2D IBL method, the CFD simulations are carried out on a 2D flat plate configuration where the VG is presented as a wall boundary condition with height, $h_{V G}$, and zero thickness.

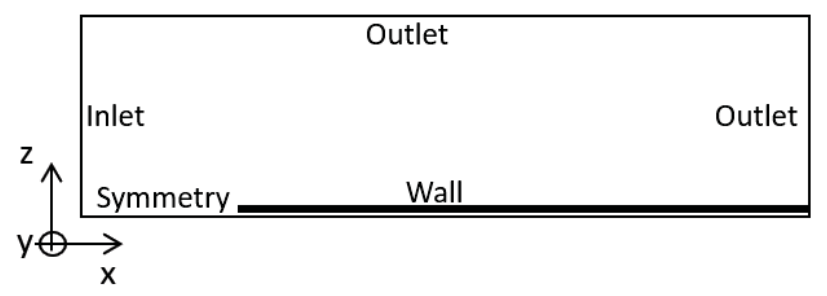

(a) Flat plate domain

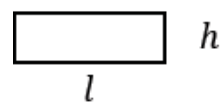

(b) VG geometry for flat plate [14].

Fig. 4 Computational domain used for flat plate simulations (a), VG geometry, $h=5 \mathrm{~mm}$ (b)

The results for the clean and the VG cases are shown in Figs. 5 to 6 . The main focus will be on the outer layer of the boundary layer away from the wall. Near wall behavior is not shown here to emphasize the behavior in the mixing region. In these figures result are presented where the locations, $x$, on the flat plate are scaled as

$$
\frac{x-x_{V G}}{h_{V G}},
$$

where $x_{V G}$ the location and $h_{V G}$ is the height of the VG.

Fig. 5a shows that the effect of the VG on the velocity profile are observed as far upstream as $-10 h_{V G}$ and extends up to approximately $100 h_{V G}$ (Fig. 5b). However, significant differences in the shape of the velocity profile are observed over a much narrower range. In Fig. 6 the vortex that is formed at the location of VG can be seen. In a clean boundary layer, the vorticity is usually maximum at the wall and the vortex strength tends to zero as we approach to the edge of the boundary layer. However, in the VG case, a new vortex starts to appear away from the wall near the VG location (about $-7 h_{V G}$ upstream) and gets dissipated as it moves downstream. The difference in vorticity magnitude near the wall (Fig. 6a) between the clean and VG is due to the fact that the boundary layer is laminar in the clean case whereas transition has already been triggered in the VG case. Identical vorticity profiles can be observed far downstream where both cases are turbulent and the effect of VG is not felt. Fig. 7a shows the eddy viscosity profile around the VG and it becomes clear that the presence of the VG causes the flow to transition to turbulent (much) earlier than the clean case $\left(v_{t}=0\right)$. The other observation to be made is that even after the flow transitions, the eddy viscosity reaches it's peak value at a higher distance from the wall compared to the clean case. This can be explained by the additional shear layers introduced (Fig. 8a) due to mixing and thus the traditional eddy viscosity profile in a boundary layer([16],[13]) is not observed anymore.

\section{Mixing layer}

As mentioned in section IV, to close the system of IBL equations a closure needed for both laminar and turbulent flow conditions are usually obtained from velocity profiles based either on extensive experimental data or theory. These velocity profiles are not valid for mixing layers as seen in Fig. 5 and different relations are needed. To obtain these new relations, the plane mixing layer theory is used. The plane mixing layer is a free shear flow and is widely studied[13],[2]. Earlier work (e.g., see, [20],[21] and [22]) in studying mixing layers have reported some correlations for parameters like momentum thickness (of the mixing layer), width of mixing region, eddy diffusivity and spreading rate under zero pressure gradient and adverse pressure gradients[21, 22]. However, most of these studies are conducted in the context of 


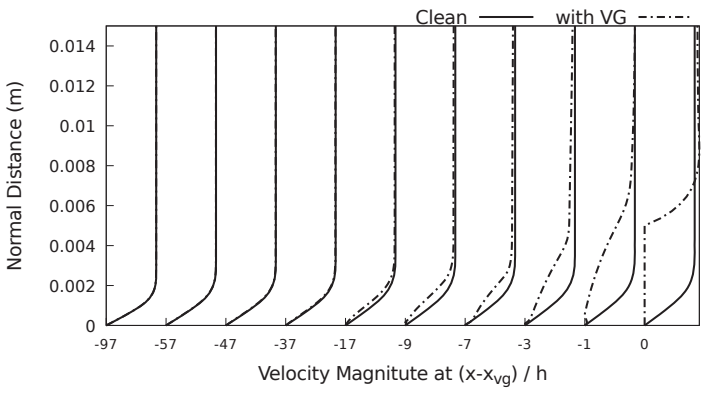

(a) around VG

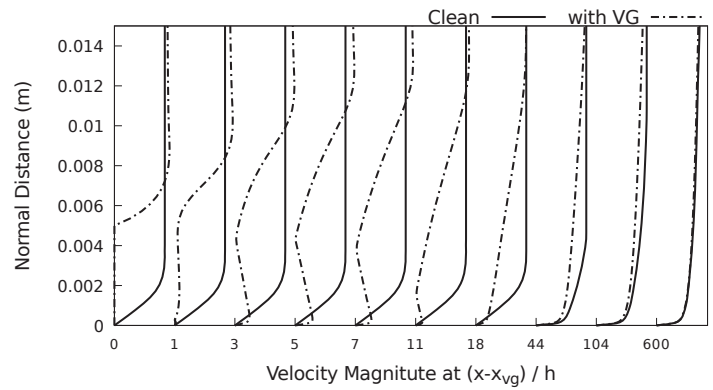

(b) downstream of VG

Fig. 5 Comparison of velocity profiles with and without VG at various locations.

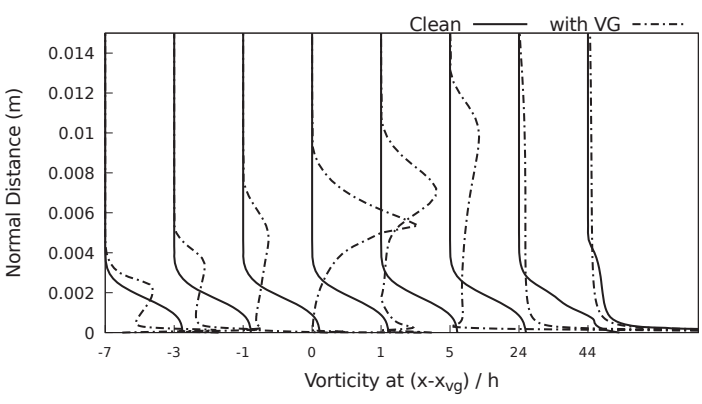

(a) around VG

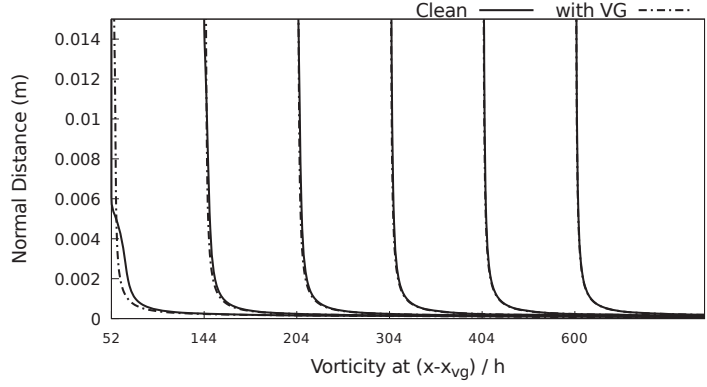

(b) downstream of VG

Fig. 6 Comparison of vorticity magnitude profiles with and without VG at various locations.

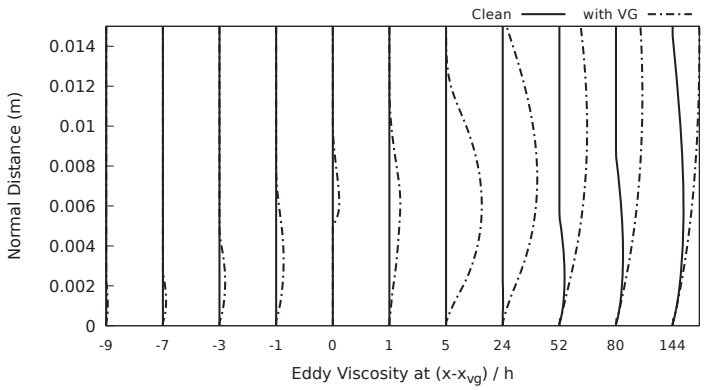

(a) around VG

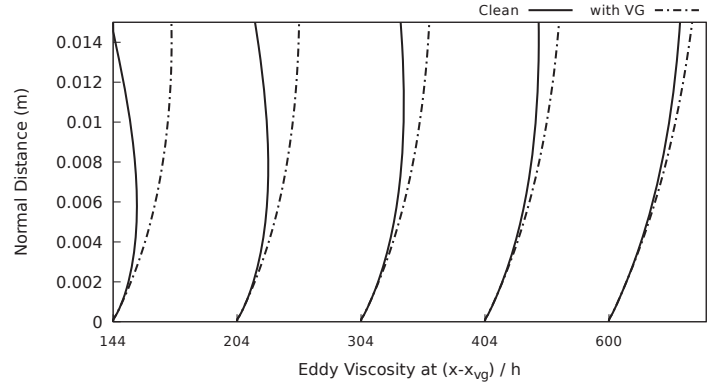

(b) downstream of VG

Fig. 7 Comparison of eddy viscosity profiles with and without VG at various locations.

free shear flows and assume that the mixing layer can spread without any constraints. As no external pressure gradient exists in the flow over a flat plate, a self similar velocity profile can be expected to form within the boundary layer region[20].

For a mixing layer ([13]), two imposed velocities, $U_{h}$ and $U_{l}$ of the two parallel streams can be defined (Fig. 3). Based on these velocities, a characteristic convection velocity, $U_{c}$, can be defined as,

$$
U_{c} \equiv \frac{1}{2}\left(U_{h}+U_{l}\right),
$$




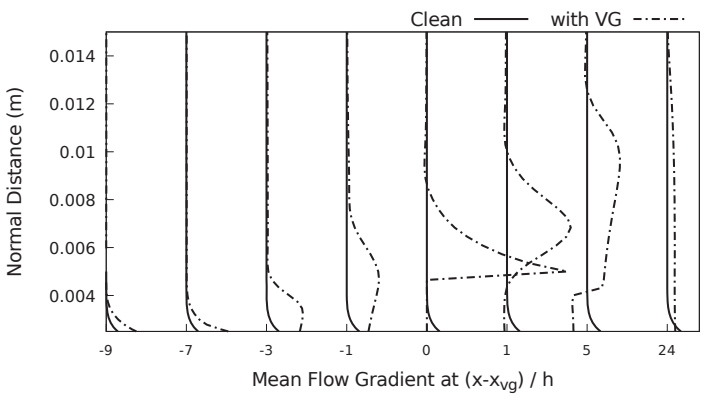

(a) around VG

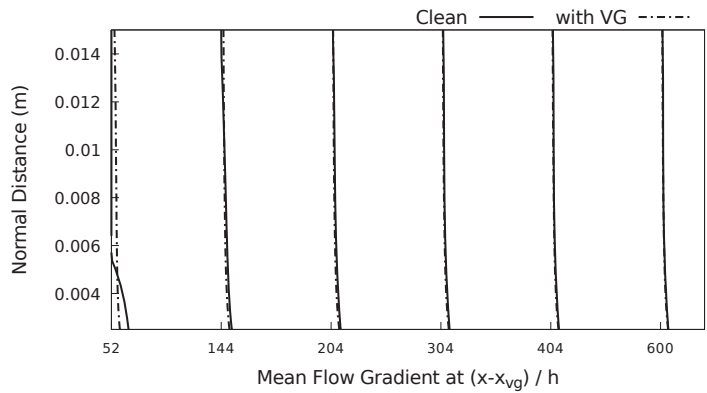

(b) downstream of VG

Fig. 8 Comparison of mean flow gradient profiles with and without VG at various locations.

and a characteristic velocity difference, $U_{s}$, as:

$$
U_{s} \equiv U_{h}-U_{l} .
$$

In the present case, the mixing layer can be assumed to form right after the VG as flow above $y=h_{V G}$ mixes with the flow below it. The characteristic velocities are however not uniform and need to be computed. These characteristic velocities are found as follows,

$$
\begin{gathered}
U_{l}=\frac{\int_{0}^{h_{V G}}(\rho u) u d y}{\int_{0}^{h_{V G}}(\rho u) d y}, \\
U_{h}=\frac{\int_{h_{V G}}^{\delta}(\rho u) u d y}{\int_{h_{V G}}^{\delta}(\rho u) d y} .
\end{gathered}
$$

In both equations, the numerator represents the momentum flux and the denominator represents the mass flux within the bounds of integration. Thus, $U_{h}$ and $U_{l}$ can be viewed as the average velocity with which the mass flux is convected in the boundary layer. The streamwise location where this integration is carried out is important and since the mixing layer is assumed to form immediately after the VG, the characteristic velocities are found at a small distance downstream $\left(\approx 3 h_{V G}\right)$ from the location of VG.

To define the characteristic width of the mixing layer based on mean velocity, $U$, a new weighting factor, $\alpha$, is introduced such that

$$
U=U_{l}+\alpha\left(U_{h}-U_{l}\right)
$$

and then width, $w(x)$, defined as

$$
w(x)=y_{\alpha=0.9}(x)-y_{\alpha=0.1}(x),
$$

and a reference lateral position is defined as

$$
\bar{w}(x)=\frac{1}{2}\left(y_{\alpha=0.9}(x)+y_{\alpha=0.1}(x)\right) .
$$

Based on these definitions, a scaled wall normal distance can be defined as,

$$
\xi=\frac{y-\bar{w}(x)-h_{V G}}{w(x)},
$$

and the scaled velocity as

$$
f(\xi)=\frac{U-U_{c}}{U_{s}} .
$$

For a plane mixing layer, the scaled velocity must be self similar. This can also be observed in the present case (Fig.9). In Fig. 9a, the scaled velocities all collapse on top of each other, however, shape of the collapsed curve is 
different from the standard mixing layer which could be due to the non uniform velocities at the start of the mixing layer. As the flow moves downstream, the width of the mixing layer grows and the presence of the wall inhibits the development leading to truncated profiles seen in Fig. 9b. The spreading of the mixing layer is seen in Fig.10a . No such self similar profile exists in the clean case (Fig. 10b).

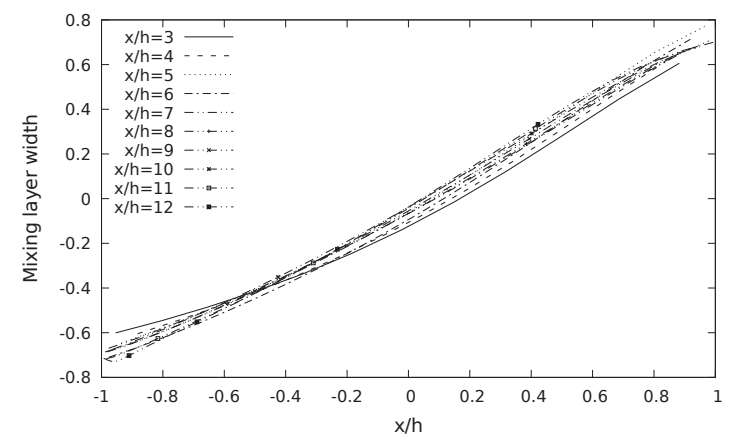

(a) Scaled velocity downstream the VG.

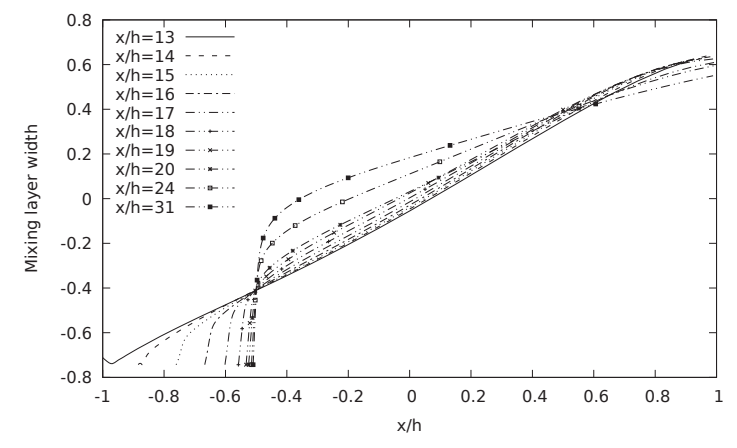

(b) Scaled velocity downstream the VG.

Fig. 9 Scaled velocity and cross stream distance.

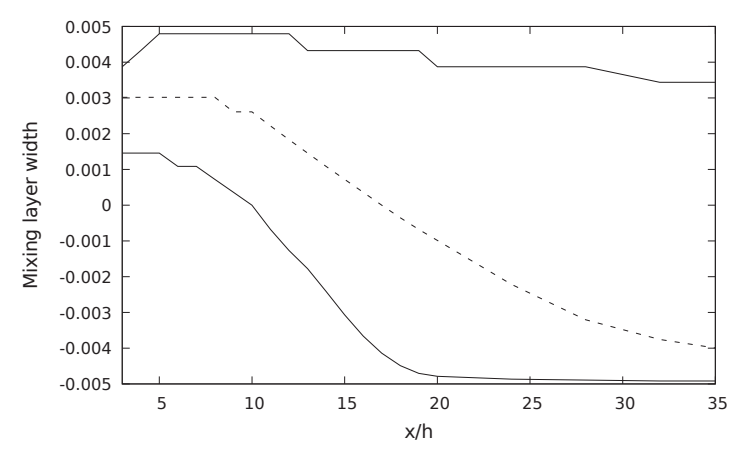

(a) Mixing layer growth.

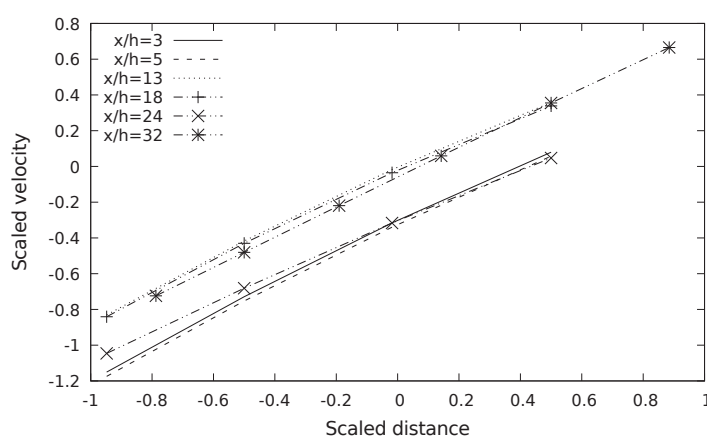

(b) Scaled velocity in a flatplate without VG.

Fig. 10 Spreading of the mixing region for the VG case (a) and the scaled velocity profiles for the clean case (b).

While such self similar profiles are not possible on airfoils due to the external gradients[20], other parametric definitions of the velocity profiles are available as shown by Sabin[22].

\section{Boundary layer modeling}

Currently, the VG model obtained for the flat plate is implemented in an in-house unsteady interactive boundary layer method[11]. As noted in section VI, more extensive work to characterize the velocity profile due to VGs are needed and in this section, a preliminary implementation of the proposed model is presented. The purpose of this implementation is to verify if the presented concept is valid.

To model the effect of VG in an IBL method, the boundary layer parameters are extracted from the CFD simulations. The edge of the boundary layer is located based on vorticity magnitude and then the displacement, momentum thickness and other boundary layer parameters are extracted by integrating the velocity profiles numerically. The extracted thicknesses are shown in Fig. 11a.

As a result of the presence of VG, the transition from laminar to turbulent flow is triggered sooner as also discussed above. However, it should be noted that this transition occurs some distance downstream of the VG and not at the location of VG. Additionally, there is a very significant increase in both momentum and displacement thicknesses due to the VG, but the shape factor in the turbulent region is actually lower than the clean flow case (Fig. 13b) . This is along expected lines as the increased mixing will increase the momentum within the boundary layer by a larger magnitude than 


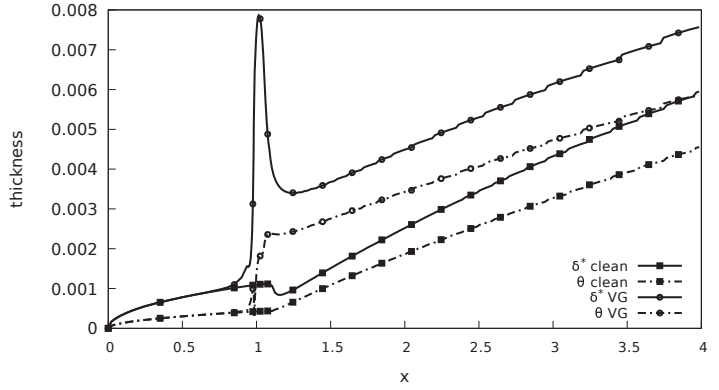

(a) CFD for clean and VG flat plate.

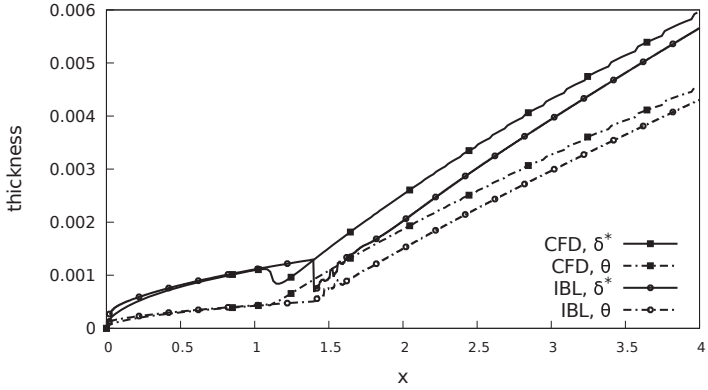

(b) CFD and IBL for clean case.

Fig. 11 Boundary layer thickness

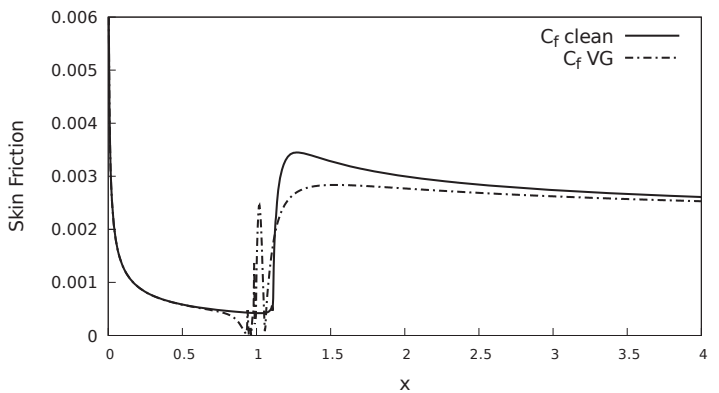

(a) Skin friction along the flat plate

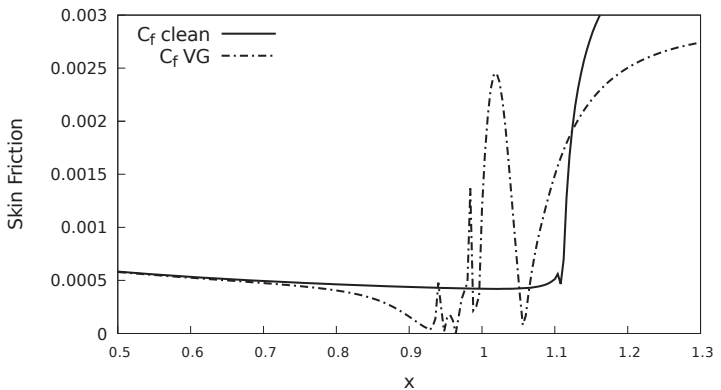

(b) Skin friction around the VG.

Fig. 12 Skin friction (magnitude).

the mass flux[2]. The difference in skin friction coefficient is shown in Figs. 12a,12b). The maximum shear stress in the VG case sees a very sharp peak (Fig. 13a) just downstream of the VG but then recovers and matches the clean case as the flow moves downstream. Interestingly, the largest difference between the shear stress coefficient is in the region between $h_{V G}$ to $30 h_{V G}$ which is also where self similar velocity profiles were observed. As the flows moves downstream, the wall inhibits the growth of the mixing layer and the $C_{f}$ and $C_{\tau}$ also recovers back to traditional boundary layer profile.

Before the VG model can be implemented in the IBL method, first a comparison between the boundary layer parameters obtained for the clean case from the CFD simulations and the IBL method is made to verify the agreement between the two methods. The two methods are in good agreement in the laminar region but the CFD simulations predict transition to occur earlier than the IBL method which leads to a difference in thickness values in the turbulent region. The IBL method uses the $e^{N}$ method $[9,10]$ to detect the location of transition and the correlation based $\mathrm{BC}$ transition model[19] is used in CFD. Subsequently, a forced transition method is used in the IBL code by triggering the transition at the same location as obtained from CFD and the non dimensional shear stress parameter, $C_{\tau}$, and skin friction coefficient, $C_{f}$, (shown in Figs. 14a and 14b) are compared. In all three cases, the skin friction in the turbulent region agrees closely. Both CFD and natural transition model of IBL display an erratic behavior in $C_{f}$ around their respective transition regions. The maximum Reynolds stress predicted by CFD is generally greater than both the IBL cases. It is somewhat more difficult to obtain the dissipation coefficient from CFD data and is not plotted here.

In order to properly introduce a VG model in IBL, closure relations need to be derived starting from the new velocity profiles. However, there is not enough data (not enough test cases simulated) to derive a reliable model yet. To test the VG model concept presented, a rudimentary model is implemented using the data extracted from CFD simulations (not necessarily building a general closure set). This gives a first impression on the validity of the described procedure. Naturally, in the follow up studies the model will be refined with more numerical and experimental data. To this end a comparison between the shape factor, $H$, in the boundary layer parameters from CFD for the clean and VG cases are made and is implemented in the IBL code. The resulting displacement and momentum thicknesses are shown in Figs. 15a and 15b and maximum shear stress profile in Fig. 15c. A forced transition method was used for this 


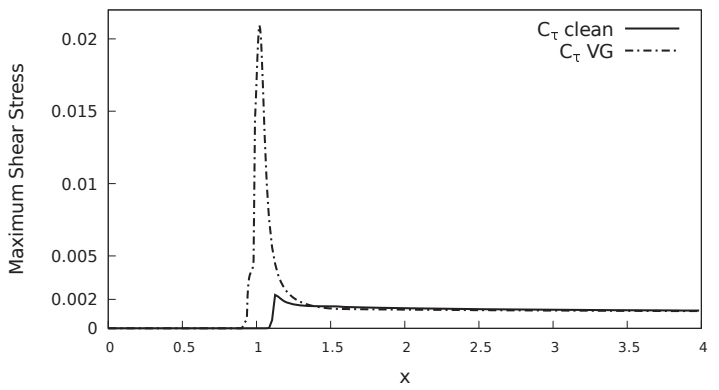

(a) Maximum shear stress coefficients.

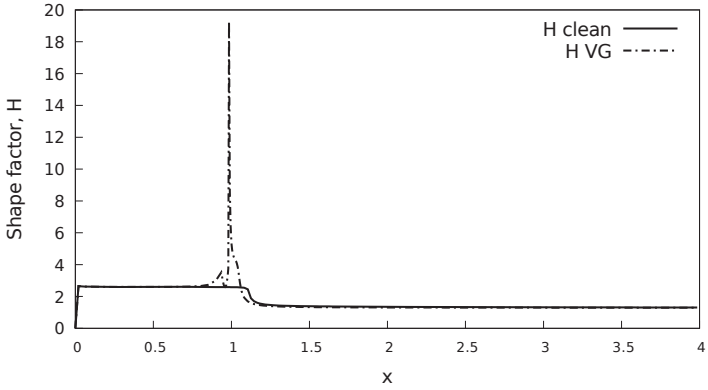

(b) Shape factor, $H$.

Fig. 13 CFD for clean and VG

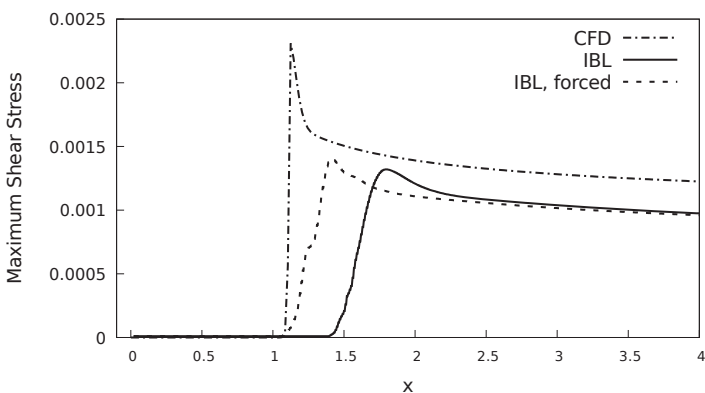

(a) Maximum shear stress

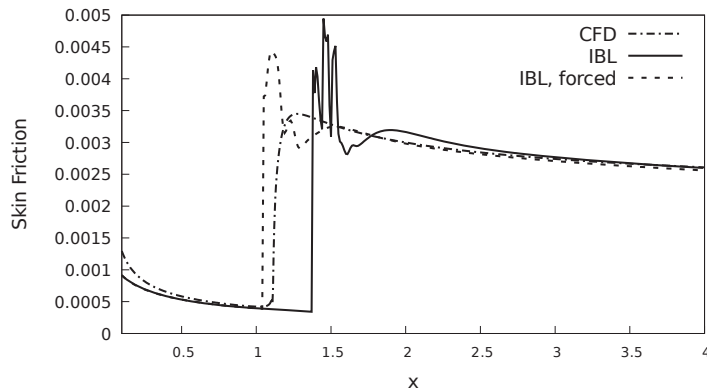

(b) Skin friction coefficient

Fig. 14 Non dimensional coefficients from CFD and IBL (natural and forced transition)

simulation. While it was not possible to match the extreme peaks observed in CFD by the IBL method, a similar trend can be observed in the behavior of shear stress and the boundary layer thicknesses. The extreme peak observed in the displacement thickness profile from CFD is most likely due to the offset in the wall boundary at the location of the VG and currently different ways to incorporate this offset is being investigated.

\section{Conclusions and future work}

In this study, it is presented that the presence of vortex generators leads to the formation of a mixing layer that is embedded in the boundary layer. However, since the mixing layer is embedded in a boundary layer, the spreading rate and other characteristics are different than a typical free shear flow. These effects can only be introduced into the IBL equations via the closure relations. To obtain such closures, a parametric family of velocity profiles is needed and such a family of profiles can be obtained based on a plane mixing layer. The velocity is self similar in flat plates in the absence of a pressure gradient but while the self similar behavior cannot be found in airfoils, parametric definitions of velocity profiles are possible.

A preliminary attempt was made at incorporating the mixing effects in the boundary layer and promising results were observed. Extensive data is needed to further verify the presence of mixing layer and for development of better closure relations.

Apart from applying the theory developed here to VGs, it can be extended to other applications that involve the presence of an additional mean flow gradient within the boundary layer. Some analogous applications would be in the analysis of atmospheric boundary layers. 


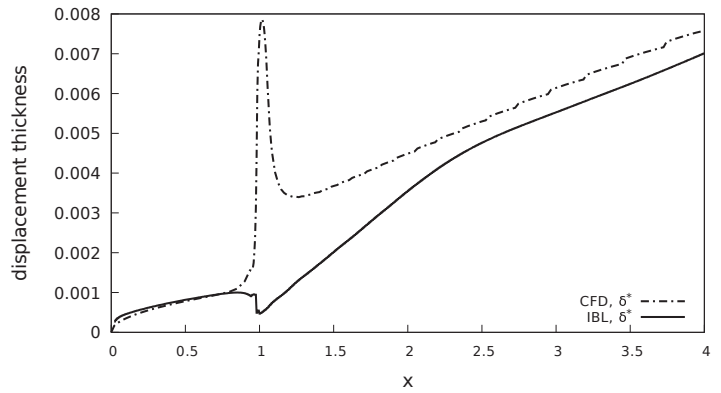

(a) Displacement thicknesses

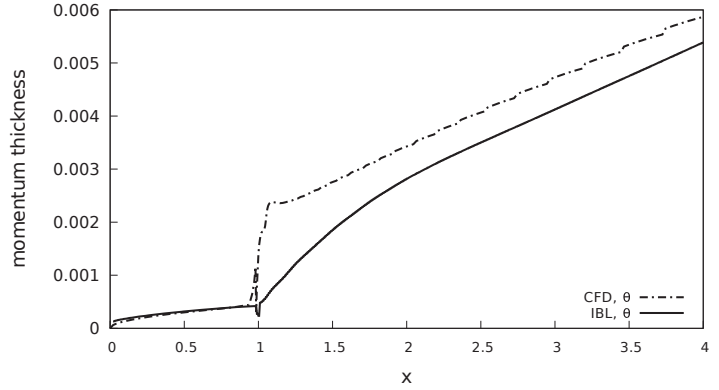

(b) Momentum thicknesses

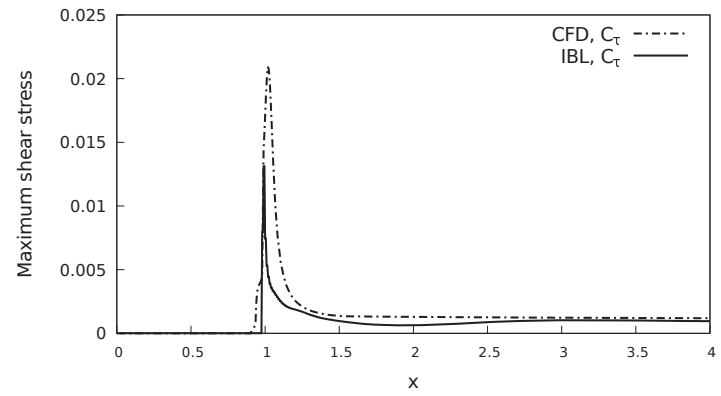

(c) Maximum shear stress

Fig. 15 A comparison of displacement and momentum thicknesses (a) and the maximum shear stress (b) obtained by CFD simulation and by the IBL method with the preliminary VG.

\section{References}

[1] Lin, J. C., "Review of research on low-profile vortex generators to control boundary-layer separation," Progress in Aerospace Sciences, Vol. 38, No. 4-5, 2002, pp. 389-420.

[2] Schubauer, G. B., and Spangenberg, W. G., "Forced mixing in boundary layers," Journal of Fluid Mechanics, Vol. 8, No. 1, 1960.

[3] Cutler, A. D., and Bradshaw, P., "Strong vortex/boundary layer interactions Part II," Experiments in Fluids, 1993.

[4] Cutler, A. D., and Bradshaw, P., "Strong vortex/boundary layer interactions Part I," Experiments in Fluids, 1993.

[5] Fuglsang, P., Antoniou, I., Dahl, K., and Aagaard Madsen, H., Wind tunnel tests of the FFA-W3-241, FFA-W3-301 and NACA 63-430 airfoils, 1998.

[6] Florentie, L., van Zuijlen, A. H., Hulshoff, S. J., and Bijl, H., "Effectiveness of Side Force Models for Flow Simulations Downstream of Vortex Generators," AIAA Journal, Vol. 55, No. 4, 2017, pp. 1373-1384. doi:10.2514/1.J055268, URL https://arc.aiaa.org/doi/10.2514/1.J055268.

[7] Fernandez, U., Velte, C. M., Rethore, P.-E., and Sorenson, N. N., "Self similarity and helical symmetry in vortex generator flow simulations," The science of making torque from wind, 2012.

[8] Burton, T., Jenkins, N., Sharpe, D., and Bossanyi, E., Wind energy handbook, John Wiley \& Sons, 2011.

[9] Drela, M., "XFOIL: An analysis and design system for low Reynolds number airfoils," Low Reynolds number aerodynamics, Springer, 1989, pp. 1-12.

[10] Ramanujam, G., Özdemir, H., and Hoeijmakers, H., "Improving airfoil drag prediction,” AIAA SciTech 2016, AIAA, 2016.

[11] Ozdemir, H., van Garrel, A., Koodly Ravishankara, A., Passalacqua, F., and Seubers, H., "Unsteady Interacting Boundary Layer Method," 35th Wind Energy Symposium, 2017, p. 2003. 
[12] Schetz, J. A., "Boundary layer analysis,” NASA STI/Recon Technical Report A, Vol. 93, 1993.

[13] Pope, S. B., Turbulent Flows, Cambridge University Press, 2000. doi:10.1017/CBO9780511840531.

[14] Waithef, K. A., “Source Term Model for Vortex Generator Vanes in a Navier-stokes Computer Code,” 2004.

[15] Matsushita, M., Murata, S., and Akamatsu, T., "Studies on Boundary-Layer Separation in Unsteady Flows Using an Itegral Method," Journal of Fluid Mechanics, Vol. 149, 1984, pp. 477-501.

[16] Drela, M., "Two-dimensional transonic aerodynamic design and analysis using the Euler equations," Ph.D. thesis, Massachusetts Institute of Technology, 1986.

[17] Palacios, F., Colono, M. R., and et al, "Stanford University Unstructured ( $\left.S U^{2}\right)$ : An open-source integrated computational environment for multi-physics simulation and design.” 51st AIAA Aerospace Sciences Meeting, AIAA, 2013.

[18] Baldacchino, D., "Experimental investigation of low profile vortex generators in a boundary layer wind tunnel," AVATAR, Task 3.1: Models for flow devices and flow control, 2015.

[19] Cakmakcioglu, S. C., Bas, O., and Kaynak, U., "A correlation-based algebraic transition model," Proceedings of the Institution of Mechanical Engineers, Part C: Journal of Mechanical Engineering Science, Vol. 232, No. 21, 2018, pp. 3915-3929.

[20] Rebello, M. R., "Analytical and Experimental Investigation of a turbulent mixing layer of different gases in a pressure gradient," Ph.D. thesis, California Institute of Technology, 1973.

[21] Konig, O., Schuluter, J., and Fiedler, H. E., "Turbulent Mixing Layer in Adverse Pressure Gradient," Progress in Fluid Flow Research: Turbulence and Applied MHD, AIAA, ????

[22] Sabin, C. M., "An analytical and experimental study of the plane, incompressible, turbulent free-shear layer with arbitrary velocity ratio and pressure gradient," Journal of Basic Engineering, Vol. 87, No. 2, 1965, pp. 421-428. 